TITLE:

\title{
Symmetry-resolved vibrational spectra of carbon K-shell photoelectron satellites in carbon monoxides: Experiment and theory
}

\section{$\operatorname{AUTHOR(S):~}$}

Ueda, K; Hoshino, M; Tanaka, T; Kitajima, M; Tanaka, H; De Fanis, A; Tamenori, Y; ... Oyagi, F; Kuramoto, K; Nakatsuji, $\mathrm{H}$

\section{CITATION:}

Ueda, K ... [et al]. Symmetry-resolved vibrational spectra of carbon K-shell photoelectron satellites in carbon monoxides: Experiment and theory. PHYSICAL REVIEW LETTERS 2005, 94(24): 243004.

ISSUE DATE:

2005-06-24

URL:

http://hdl.handle.net/2433/39926

RIGHT:

Copyright 2005 American Physical Society 


\title{
Symmetry-Resolved Vibrational Spectra of Carbon $K$-Shell Photoelectron Satellites in Carbon Monoxides: Experiment and Theory
}

\author{
K. Ueda,${ }^{1, *}$ M. Hoshino, ${ }^{2}$ T. Tanaka, ${ }^{3}$ M. Kitajima,${ }^{3}$ H. Tanaka, ${ }^{3}$ A. De Fanis, ${ }^{4}$ Y. Tamenori, ${ }^{4}$ M. Ehara, ${ }^{5}$ F. Oyagi, ${ }^{5}$ \\ K. Kuramoto, ${ }^{5}$ and H. Nakatsuji ${ }^{5}$ \\ ${ }^{1}$ Institute of Multidisciplinary Research for Advanced Materials, Tohoku University, Sendai 980-8577, Japan \\ ${ }^{2}$ RIKEN, Wako, Saitama 351-0198, Japan \\ ${ }^{3}$ Department of Physics, Sophia University, Chiyoda-ku, Tokyo 102-8554, Japan \\ ${ }^{4}$ Japan Synchrotron Radiation Research Institute, Sayo-gun, Hyogo 679-5198, Japan \\ ${ }^{5}$ Department of Synthetic Chemistry and Biological Chemistry, Graduate School of Engineering, Kyoto University, \\ Kyoto-Daigaku-Katsura, Kyoto 615-8510, Japan
}

(Received 10 March 2005; published 23 June 2005)

\begin{abstract}
We have carried out angle-resolved measurement for the $\mathrm{C} 1 s$ photoelectron shakeup satellites of gaseous $\mathrm{CO}$ molecules, at unprecedented energy resolution, and resolved the vibrational structures for one ${ }^{2} \Sigma^{+}$and two ${ }^{2} \Pi$ bands. The present $a b$ initio calculations for the potential curves of these shakeup satellite states and the relevant Franck-Condon analysis well reproduce the vibrational structures of these bands and confirm the assignments.
\end{abstract}

DOI: 10.1103/PhysRevLett.94.243004

Properties of molecules depend on the nuclear conformation. Electronically excited states often have individual stable geometries that are different from the ground state and thus may reveal new properties. One of the aims of molecular spectroscopy is to extract the information of the conformation of the ground and electronically excited states. Photoexcitation causes a sudden redistribution of electric charge. The nuclei cannot adapt to it on the same time scale and is considered stationary during an electronic transition, which is well known as the Franck-Condon (FC) principle. In consequence, the final state is often left vibrationally excited. Thus, one can obtain the information about the stable geometry of the electronically excited state, analyzing the vibrational population left in the excited state on the basis of the FC principle [1].

High photon fluxes with very narrow photon bandwidths via high-resolution soft $\mathrm{x}$-ray monochromators installed in high-brilliance synchrotron radiation light sources invoked a renewal of interest in core-level photoelectron spectroscopy [2-4]. One can measure vibrational structures in the core-level photoelectron spectrum and discuss the stable geometry of the core-ionized states. In this Letter, we demonstrate that the vibrational structures can be observed even for core-level photoelectron satellites and thus the conformations of the satellite states can be discussed in the light of the observed vibrational structure.

It is known that the photoelectron satellites provide evidence pointed to the breakdown of the independentparticle (frozen orbital) picture of electronic structure and thus are often called correlation satellites [5,6]. Correlation satellites in atomic ionization spectra have been well studied using synchrotron radiation as a tunable light source (see, i.e., [7-9]). It turned out that the satellites can be classified into two groups phenomenologically [7]. The first group includes the satellites whose excitation cross sections relative to the single-hole ionization cross
PACS numbers: 33.60.Fy, 33.70.Ca, 33.80.Eh

section stay relatively constant while the second group includes those whose excitation cross sections sharply decrease with an increase in energy. These features are also seen in correlation satellites in molecular ionization spectra $[6,10]$. The two different types of energy dependence have been attributed to the two lowest-order correlation terms, often called the direct and conjugate shakeup terms $[9,10]$. In the direct shakeup term, the dipole photoemission from the core orbital (bound-free dipole integral) is accompanied by the monopole excitation of the valence electron (bound-bound overlap integral). In the conjugate shakeup term, the dipole excitation of the core electron to an unoccupied molecular orbital (bound-bound dipole integral) is accompanied by the monopole ionization of the valence electron (bound-free overlap integral): the conjugate shakeup contribution is significant near the ionization threshold region and decreases rapidly with the increase in energy $[6,10]$. It should be noted that the direct and conjugate channels may have the same final state and thus the interference may take place [11]. In spite of the extensive studies of the molecular photoelectron satellites, however, there have been no report on vibrationally resolved satellite bands to our knowledge.

The molecule concerned here is carbon monoxide (CO). The CO molecule has a ground state electronic configuration of

$$
1 \sigma^{2} 2 \sigma^{2} 3 \sigma^{2} 4 \sigma^{2} 1 \pi^{4} 5 \sigma^{2} ; 2 \pi^{* 0} 6 \sigma^{* 0} X^{1} \Sigma^{+},
$$

where $1 \sigma$ and $2 \sigma$ denote $\mathrm{O} 1 s$ and $\mathrm{C} 1 s$ core orbitals, respectively. When $\mathrm{C} 1 \mathrm{~s}$ photoemission takes place, a $1 \pi$ electron can be shaken up to an unoccupied $2 \pi^{*}$ orbital [12]. The final shakeup states are $2 \sigma^{-1} 1 \pi^{-1} 2 \pi^{* 2} \Sigma^{+} S 1$ and $S 0$, where $S 1$ and $S 0$ are triplet- and singlet-coupled $\pi-\pi^{*}$ excitations, respectively. These two states are located 8.3 and $14.9 \mathrm{eV}$ (vertical) above the $\mathrm{C} 1 s^{-1}$ 
single-hole (main) state [13]. Ab initio calculations elucidated that a significant enhancement of the first $\pi-\pi^{*} S 1$ satellite in $\mathrm{C} 1 s$ photoemission of $\mathrm{CO}$ [14] was a result of constructive interference between the direct and conjugate channels [11].

We note that, although the direct shakeup leads only to the final ionic states of ${ }^{2} \Sigma^{+}$symmetry, the conjugate shakeup may lead to final ionic states of different symmetries such as $2 \sigma^{-1} 5 \sigma^{-1} 2 \pi^{* 2} \Pi$. According to the calculations by Angonoa et al. by means of the fourth-order Green's function method [15], referred to as ADC(4) calculations, energies (vertical) of the two ${ }^{2} \Pi$ states are 8.44 and $9.20 \mathrm{eV}$ above the $\mathrm{C} 1 \mathrm{~s}^{-1}$ single-hole state while that of $2 \sigma^{-1} 1 \pi^{-1} 2 \pi^{* 2} \Sigma^{+} S 1$ state is $9.11 \mathrm{eV}$. Thus the vibrational bands arising from the transitions to the two ${ }^{2} \Pi$ final states may be overlapped with that of the ${ }^{2} \Sigma^{+} S 1$ band. In the experimental spectrum $[11,14]$, however, these two ${ }^{2} \Pi$ states have not been identified so far.

In the present study, we demonstrate that one can identify the shakeup transitions to these two ${ }^{2} \Pi$ states as well as to the ${ }^{2} \Sigma^{+}$state: these three bands are, indeed, energetically overlapped but can be separable in the angleresolved photoelectron spectra. Furthermore, our unprecedented energy resolution also allows us to extract the individual vibrational structures of these three states and to provide key information about the potential curves of these three states. Franck-Condon analysis based on the $a b$ initio potential curves, calculated by means of the symmetry-adapted cluster-expansion configurationinteraction (SAC-CI) method [16], well reproduces the three vibrationally resolved satellite bands and confirms the assignments of these newly observed bands as well as the validity of the calculated potential curves.

The experiment was carried out at the high-resolution photochemistry beam line 27SU [17,18] in SPring-8, Japan. The light source of this beam line is a figure- 8 undulator [19]. The first-order harmonic light generated by this undulator has a horizontal linear polarization while the so-called 0.5 th order harmonic light has a vertical polarization. The electron spectroscopy apparatus was described elsewhere [20] and only some specific features are summarized here. The apparatus consists of an electron energy analyzer (Gammadata-Scienta SES-2002), a gas cell (GC-50), and a differentially pumped main chamber. The lens axis of the analyzer is set in a horizontal direction so that the entrance slit of the analyzer is parallel to the photon direction. The whole system sits on an adjustable stage so that the source point of the analyzer can be adjusted easily relative to the fixed beam position. For the angle-resolved electron spectroscopy, the spectrometer is fixed in this state while switching the direction of the electric vector between horizontal and vertical. The degree of linear polarization was measured by observing the Ne $2 s$ and $2 p$ photolines. It was found to be larger than 0.98 for the current optical settings, thus enabling the assumption that all photons are completely polarized.
Figure 1 illustrates the lowest satellite bands recorded at two photon energies 310 and $330 \mathrm{eV}$ at two angles $0^{\circ}$ and $90^{\circ}$ relative to the polarization vector. The overall resolution is $\sim 50 \mathrm{meV}$. In the $90^{\circ}$ spectrum at $310 \mathrm{eV}$, we can see two vibrational bands. Looking at the $0^{\circ}$ spectrum carefully, we notice that there are some peaks which are not seen at $90^{\circ}$ spectrum. When the photon energy increases by $20 \mathrm{eV}$, we see a dramatic change in the spectra. The change is due to a rapid intensity drop of the $90^{\circ}$ spectrum and of the corresponding band in the $0^{\circ}$ spectrum. From these spectra, it is clear that there are at least three electronic states involved.

To understand the observed energy dependence and angular distribution of the satellite spectra, we briefly look into the direct and conjugate terms. The dominant terms in the dipole transition moments concerned can schematically be written as

$$
\begin{aligned}
& D\left(2 \sigma^{-1} 1 \pi^{-1} 2 \pi^{*} \epsilon \sigma\right) \sim\left\langle\epsilon \sigma^{*}|r| 2 \sigma\right\rangle\left\langle 2 \pi^{*} \mid 1 \pi\right\rangle, \\
& D\left(2 \sigma^{-1} 1 \pi^{-1} 2 \pi^{*} \epsilon \pi\right) \sim\langle\epsilon \pi|r| 2 \sigma\rangle\left\langle 2 \pi^{*} \mid 1 \pi\right\rangle \\
& \quad+\left\langle 2 \pi^{*}|r| 2 \sigma\right\rangle\langle\epsilon \pi \mid 1 \pi\rangle,
\end{aligned}
$$$$
D\left(2 \sigma^{-1} 5 \sigma^{-1} 2 \pi^{*} \epsilon \sigma\right) \sim\left\langle 2 \pi^{*}|r| 2 \sigma\right\rangle\langle\epsilon \sigma \mid 5 \sigma\rangle .
$$

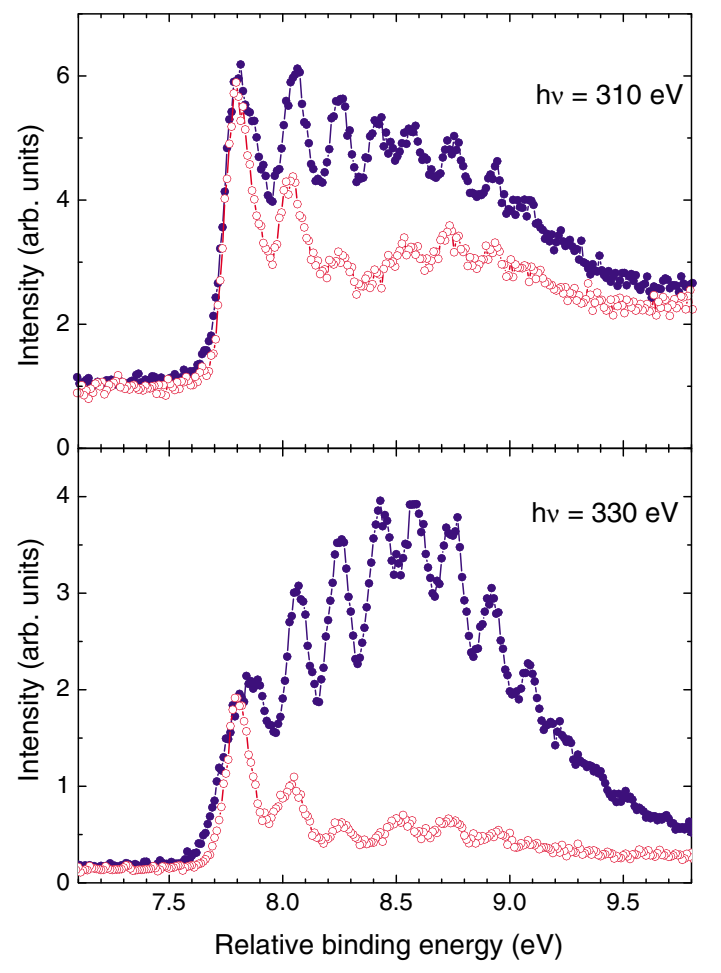

FIG. 1 (color online). C $1 s$ photoelectron satellite spectra of $\mathrm{CO}$ recorded at photon energies of $310 \mathrm{eV}$ (upper panel) and $330 \mathrm{eV}$ (lower panel), at $0^{\circ}$ (blue closed circles) and $90^{\circ}$ (red open circles) relative to the polarization vector, plotted as a function of the binding energy relative to the $\mathrm{C} 1 s$ single-hole state. 
From Eq. (1), it is clear that the $\Sigma-\Sigma$ transition, in which the dipole moment is parallel to the molecular axis, leading to the ionic final state $2 \sigma^{-1} 1 \pi^{-1} 2 \pi^{* 2} \Sigma^{+} S 1$, is dominated by the direct shakeup contribution (bound-free dipole integral and bound-bound overlap integral). From Eq. (3), it is clear the transition leading to the ionic final state $2 \sigma^{-1} 5 \sigma^{-1} 2 \pi^{* 2} \Pi$ is dominated by the conjugate shakeup contribution (bound-free overlap integral and bound-bound dipole integral) of $\Sigma-\Pi$ transition symmetry, in which the dipole moment is perpendicular to the molecular axis. One can see from Eq. (2) both the direct and the conjugate terms contribute to the $\Sigma$ - $\Pi$ transition leading to the ionic final state $2 \sigma^{-1} 1 \pi^{-1} 2 \pi^{* 2} \Sigma^{+} S 1$ and can cause interference as pointed out by Schirmer et al. [11]. It should also be noted that the conjugate contributions become negligible at high energy because of the sharp drop of the bound-free overlap integral. At this limit, the direct shakeup satellite band mimics the mainline both in the excitation cross section and in the asymmetry parameter $\beta$ of the electron emission: $\beta$ approaches the limiting value two.

Using the above described characteristics of direct and conjugate shakeup contributions, we can assign the observed three vibrational bands in Fig. 1. The band that appears in the $0^{\circ}$ spectrum at $330 \mathrm{eV}$ nearly disappears in the $90^{\circ}$ spectrum, exhibiting a very strong anisotropy with $\beta$ close to 2 . Thus we can conclude that this band is dominated by the direct shakeup contribution and thus is assigned to the transition leading to the ionic final state $2 \sigma^{-1} 1 \pi^{-1} 2 \pi^{* 2} \Sigma^{+} S 1$. The two bands that appear in the $90^{\circ}$ spectrum at $330 \mathrm{eV}$, on the other hand, can be attributed to the conjugate shakeup contributions and thus are assigned to the transitions leading to $2 \sigma^{-1} 5 \sigma^{-1} 2 \pi^{* 2} \Pi$. These assignments are consistent with the $a b$ initio energies by means of $\mathrm{ADC}(4)$ : the observed energies (vertical), $8.5 \mathrm{eV}$ for ${ }^{2} \Sigma^{+}$and 7.9 and $8.7 \mathrm{eV}$ for ${ }^{2} \Pi$ agree reasonably with the calculated state energies (vertical), $9.11 \mathrm{eV}$ for ${ }^{2} \Sigma^{+} S 1$ and 8.24 and $9.20 \mathrm{eV}$ for ${ }^{2} \Pi$.

The vibrational structure observed in each band includes the information about the potential curve of the relevant satellite state. However, neither the contribution from the ${ }^{2} \Sigma^{+} S 1$ band to the $90^{\circ}$ spectrum nor the ${ }^{2} \Pi$ bands to the $0^{\circ}$ spectrum may be negligible, and also $\beta$ for the individual vibrational components may not be the same. Thus, instead of trying to extract the potential parameters from the spectra we decided to perform ab initio calculations for the relevant potential curves by means of the SAC-CI method [16].

We have calculated the potential energy curves of the ground and $\mathrm{C} 1 s$ shakeup satellite states, ${ }^{2} \Sigma^{+},{ }^{2} \Pi,{ }^{2} \Delta$, and ${ }^{2} \Sigma^{-}$states, in the region of $R=0.9-1.7 \AA$. The basis sets were triple zeta $(10 s 6 p) /[5 s 3 p]$ Gaussian-type orbitals of Ahlrichs [21] with two polarization $d$ functions. In the SAC-CI calculation, single to quadruple $R$ operators were included. For the perturbation selection of operators, reference functions were chosen from the CI vectors with single, double, and triple excitations. The thresholds of the linked terms were $1.0 \times 10^{-6}$ and $5.0 \times 10^{-8}$ for the ground and inner-shell ionized states, respectively. The SAC/SAC-CI calculations were executed with the GAUSSIAN03 suite of programs with the modifications for calculating the inner-shell ionized states [22].

The potential energy curves of the ground and $\mathrm{C} 1 \mathrm{~s}$ shakeup satellite states were shown in Fig. 2. For the ground state, the calculated equilibrium distance $r_{e}$ and the vibrational frequency $\omega_{e}$ of the ground state are $1.126 \AA$ and $270 \mathrm{meV}$. These are in excellent agreement with the experimental values of $1.128 \AA$ and $269 \mathrm{meV}$, respectively [1]. The geometry relaxation by the shakeup ionization is large for the ${ }^{2} \Sigma^{+}$state and the calculated value of $r_{e}$ is $1.292 \AA$, i.e., $0.166 \AA$ longer than the ground state. The geometry relaxation for the two ${ }^{2} \Pi$ states is smaller than ${ }^{2} \Sigma^{+}: r_{e}=1.168$ and $1.194 \AA$ for the lower and higher ${ }^{2} \Pi$ states, respectively. The avoided crossing occurs between two ${ }^{2} \Pi$ states at $r_{e} \simeq 1.45 \AA$. The ${ }^{2} \Delta$ and ${ }^{2} \Sigma^{-}$states are almost degenerate and their potential energy curves are almost parallel to that of the ${ }^{2} \Sigma^{+}$state. The $r_{e}$ values are 1.309 and $1.320 \AA$ for $^{2} \Delta$ and ${ }^{2} \Sigma^{-}$, respectively. The reason for the similarity of the three states ${ }^{2} \Sigma^{+},{ }^{2} \Delta$, and ${ }^{2} \Sigma^{-}$can be found in the fact that all these states have the same configuration $2 \sigma^{-1} 1 \pi^{-1} 2 \pi^{*}$.

For calculating the vibrational spectrum, vibrational wave functions and the FC factors were obtained by the grid method, in which the Lanczos algorithm was adapted for the diagonalization. The SAC-CI theoretical spectra thus obtained are compared with the experimental photoelectron satellite spectra for the $\Sigma^{+}$and $\Pi$ symmetries in Fig. 3. Here the experimental $\Pi$ spectrum was approximated by the $90^{\circ}$ spectrum in Fig. 1. The experimental $\Sigma$ spectrum was approximated by $I(0)-c \times I(90)$, where $I(0)$ and $I(90)$ are spectral distributions of the $0^{\circ}$ and $90^{\circ}$

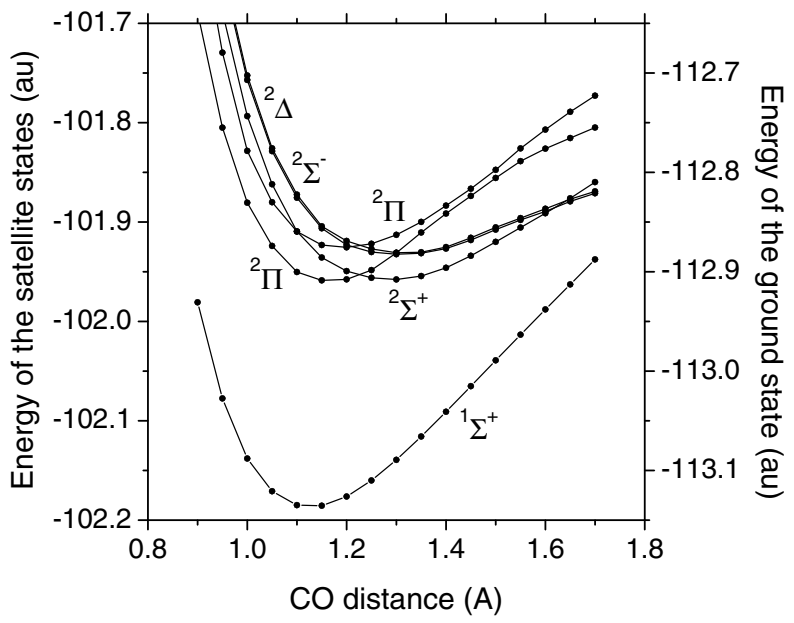

FIG. 2. Potential energy curves of the ground and C $1 s$ shakeup satellite ${ }^{2} \Sigma^{+}$and ${ }^{2} \Pi$ states of $\mathrm{CO}$ calculated by the SAC-CI method. 


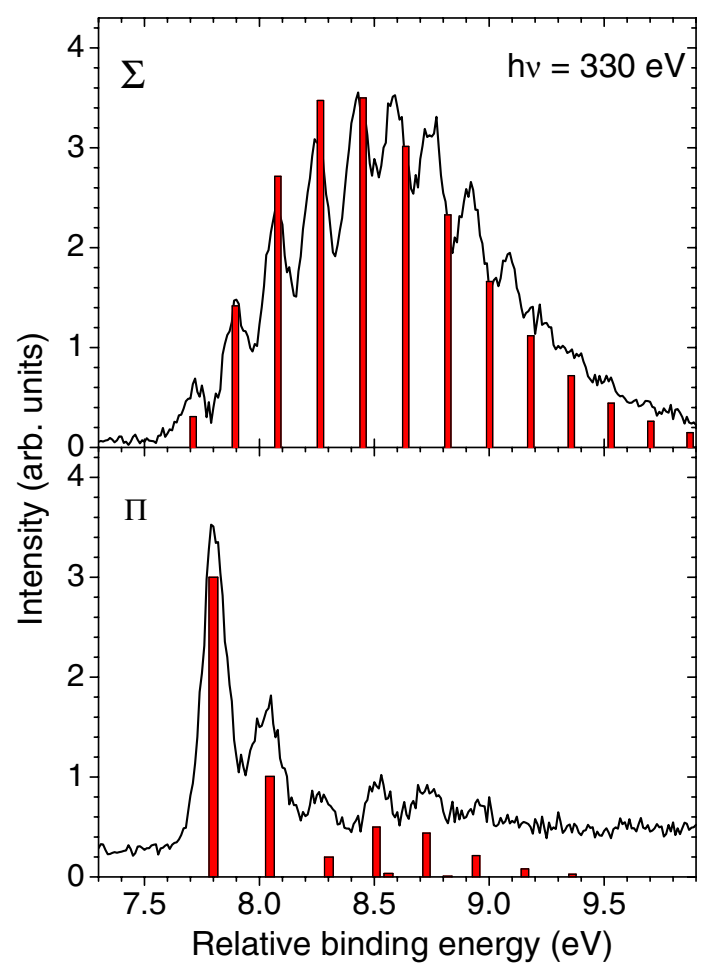

FIG. 3 (color online). C $1 s$ Satellite bands for the transitions to $2 \sigma^{-1} 1 \pi^{-1} 2 \pi^{* 2} \Sigma^{+}$(upper panel) and to $2 \sigma^{-1} 5 \sigma^{-1} 2 \pi^{* 2} \Pi$ (lower panel): comparison between experiment and theory.

spectra, respectively, and $c$ is the parameter that represents the contribution from the $\Pi$ component to the $I(0)$ spectrum; here it was selected to be $c=0.4$ so that the vibrational distribution of the $\Sigma$ spectrum is close to the Poissonian. The theoretical spectra were shifted down by $-0.23 \mathrm{eV}$ for $\Sigma^{+}$, by $-0.12 \mathrm{eV}$ for lower $\Pi$, and by $-0.32 \mathrm{eV}$ for higher $\Pi$. The relative intensities of the bands were adjusted so that the theoretical spectra gave overall agreement with experimental spectra: the vibrational intensity ratios were determined by the FC factors. The theoretical spectrum for $\Sigma^{+}$has the maximum intensity at $v=4$, reproducing the experimental spectrum well. The activation of the high vibrational states is due to the large relaxation, i.e., the elongation of the bond length by $0.17 \AA$. Deviations between the theoretical and experimental spectra in the higher vibrational members can be attributed to the errors of the calculated potential curves in the large nuclear distances. The theoretical spectra for both $\Pi$ bands have a maximum intensity at $v=0$ as a result of the small relaxation, i.e., the elongation of less than $0.1 \AA$. The theoretical spectra for the $\Sigma^{-}$and $\Delta$ symmetries fall into a higher energy region peaked at $\sim 9.6 \mathrm{eV}$. There is no trace of these bands in Fig. 1.

In conclusion, we have observed, for the first time, vibrationally resolved C $1 s$ photoelectron satellites of gaseous $\mathrm{CO}$ molecules. The angle-resolved spectra allow us to assign the observed bands to one $2 \sigma^{-1} 1 \pi^{-1} 2 \pi^{* 2} \Sigma^{+}$ state and two $2 \sigma^{-1} 5 \sigma^{-1} 2 \pi^{* 2} \Pi$ states. The vibrational excitation of these bands are well interpreted by the present $a b$ initio calculations. Excellent agreement between observed and calculated vibrational spectra supports the validity of the present $a b$ initio potential curves of these shakeup satellite states.

The experiment was carried out with the approval of the SPring-8 program review committee. This study was supported by a Grant for Creative Scientific Research from the Ministry of Education, Science, Culture, and Sports of Japan and by Grants-in-Aid for Scientific Research from the Japanese Society for the Promotion of Science.

*Electronic address: ueda@tagen.tohoku.ac.jp

[1] G. Herzberg, Molecular Spectra and Molecular Structure I. Spectra of Diatomic Molecules (D. Van Nostrand, London, 1967).

[2] K. J. Borve, L. J. Sæthre, T. D. Thomas, T. X. Carroll, N. Berrah, J.D. Bozek, and E. Kukk, Phys. Rev. A 63, 012506 (2001).

[3] T. Karlsen, L. J. Sæthre, K. J. Borve, N. Berrah, J. D. Bozek, T.X. Carroll, and T. D. Thomas, J. Phys. Chem. A 105, 7700 (2001).

[4] U. Hergenhahn, J. Phys. B 37, R89 (2004).

[5] L.S. Cederbaum, W. Domcke, J. Schirmer, and W. von Niessen, Adv. Chem. Phys. 65, 115 (1986).

[6] A. D. O. Bawagan and E. R. Davidson, Adv. Chem. Phys. 110, 215 (1999).

[7] U. Becker and D. A. Shirley, Phys. Scr. T31, 56 (1990).

[8] B. Langer, J. Viefhaus, O. Hemmers, A. Menzel, R. Wehlitz, and U. Becker, Phys. Rev. A 43, R1652 (1991).

[9] V. Schmidt, Rep. Prog. Phys. 55, 1483 (1992).

[10] L. Ungier and T.D. Thomas, Phys. Rev. Lett. 53, 435 (1984).

[11] J. Schirmer, M. Braunstein, and V. McKoy, Phys. Rev. A 44, 5762 (1991).

[12] T. D. Thomas, Phys. Rev. Lett. 52, 417 (1984).

[13] J. Schirmer, G. Angonoa, S. Svensson, D. Nordfors, and U. Gelius, J. Phys. B 20, 6031 (1987).

[14] A. Reimer et al., Phys. Rev. Lett. 57, 1707 (1986).

[15] G. Angonoa, I. Walter, and J. Schirmer, J. Chem. Phys. 87, 6789 (1987).

[16] H. Nakatsuji, in Computational Chemistry, Review of Current Trends Vol. 2 (World Scientific, Singapore, 1997), p. 62.

[17] H. Ohashi et al., Nucl. Instrum. Methods Phys. Res., Sect. A 467-468, 529 (2001).

[18] H. Ohashi et al., Nucl. Instrum. Methods Phys. Res., Sect. A 467-468, 533 (2001).

[19] T. Tanaka and H. Kitamura, J. Synchrotron Radiat. 3, 47 (1996).

[20] Y. Shimizu et al., J. Electron Spectrosc. Relat. Phenom. 114-116, 63 (2001).

[21] A. Schaefer, C. Huber, and R. Ahlrichs, J. Chem. Phys. 100, 5829 (1994).

[22] M. J. Frisch et al., GAUSSIAN03, Gaussian Inc., Pittsburgh, PA, 2003. 